PHYSICAL REVIEW B 68, 029901(E) (2003)

\title{
Erratum: Orbital ordering and exchange interaction in the manganites [Phys. Rev. B 64, 094433 (2001)]
}

Hakim Meskine, Harald König, and Sashi Satpathy

(Received 29 April 2003; published 2 July 2003)

DOI: 10.1103/PhysRevB.68.029901

PACS number(s): 75.30.Et, 75.47.Gk, 72.80.Ga, 99.10.Cd

In Fig. 10, the labels " $J_{a b}$ " and " $J_{c}$ " were interchanged. The correct figure along with the original caption appears below.

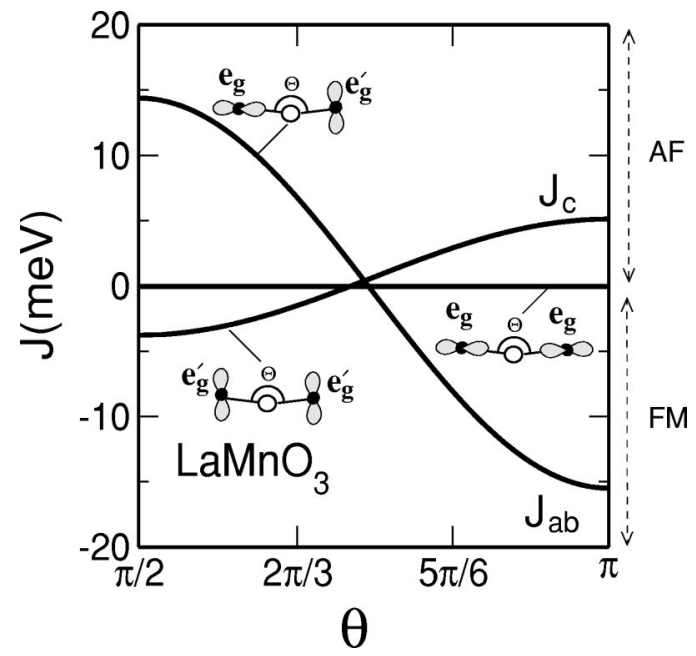

FIG. 10. Exchange interaction in $\mathrm{LaMnO}_{3}$ with the full Hamiltonian, i.e., with $J_{H}=1 \mathrm{eV}$ and with $t_{2 g}$ hopping included. The intraplane and interplane exchanges, $J_{a b}$ and $J_{c}$, correspond to the $e_{g}-e_{g}^{\prime}$ and $e_{g}^{\prime}-e_{g}^{\prime}$ orbital orientations, respectively. 